\title{
NIR high-resolution imaging and radiative transfer modeling of the Frosty Leo nebula
}

\author{
K. Murakawa ${ }^{1}$, K. Ohnaka ${ }^{1}$, T. Driebe ${ }^{1}$, K.-H. Hofmann ${ }^{1}$, \\ D. Schertl ${ }^{1}$, S. Oya ${ }^{2}$, and G. Weigelt ${ }^{1}$ \\ ${ }^{1}$ Max-Planck-Institut für Radioastronomie, Auf dem Hügel 69, D-53121 Bonn, Germany \\ email: murakawa@mpifr-bonn.mpg.de \\ ${ }^{2}$ Subaru telescope, 650 North A'ohoku Place, Hilo, HI 96720, USA
}

\begin{abstract}
We present a $K^{\prime}$-band speckle image and $H K$-band polarimetric images of the protoplanetary nebula Frosty Leo obtained using the $6 \mathrm{~m}$ SAO telescope and the $8 \mathrm{~m}$ Subaru telescope, respectively. Our speckle image revealed clumpy structures in the hourglass-like bipolar nebula. The polarimetric data, for the first time, detected an elongated region with small polarizations and polarization vector alignment on the east side of the central star. We have performed radiative transfer calculations to model the dust shell of Frosty Leo. We found that micron-size grains in the equatorial dense region and small grains in the bipolar lobes are required to explain the total intensity images, the polarization images, and the spectral energy distribution.
\end{abstract}

Keywords. speckle imaging, polarimetry, radiative transfer modeling, Frosty Leo, PPN

\section{Introduction}

Frosty Leo is a well-studied oxygen-rich proto-planetary nebula (PPN). The deep water ice absorption feature at $3.1 \mu \mathrm{m}\left(\tau_{3.1 \mu \mathrm{m}} \sim 3.3\right)$ was detected in the $L$-band spectrum (Rouan et al. 1988). Omont et al. (1990) concluded from a 35-68 $\mu \mathrm{m}$ spectrum and a simple modeling that the water ice is clearly crystalline, the emissivity index of the silicates is closer to that of crystalline silicates than to amorphous ones, and the volumes of ice and of silicates are comparable. Near-infrared imaging with adaptive optics have revealed an hourglass-like bipolar feature with a bright central star (e.g. Roddier et al. 1995). Optical to near-infrared imaging polarimetry have shown a centrosymmetric polarization pattern in the bipolar lobes and a polarization disk between them, implying that the lobes are illuminated by the central star and are split by a dense disk in the equatorial plane (Scarrott \& Scarrott 1990; Dougados et al. 1990). While it appears to be axisymmetric within $5^{\prime \prime}$ from the central star, at $\sim 10^{\prime \prime}$ several complex features such as ansae and jets have been detected (Sahai et al. 2000).

\section{Observations}

We performed $K^{\prime}$-band speckle imaging using the $6 \mathrm{~m}$ SAO telescope on March 11, 2001. The resulting image (Fig. 1 left) was reconstructed from the 581 speckle interferograms by means of the speckle masking bispectrum method (Weigelt 1977). We also obtained $H K$-band polarimetric images using the high-resolution near-infrared camera CIAO with the 36 element adaptive optics on the $8 \mathrm{~m}$ Subaru telescope on January 10, 2004. Figure 1 right shows a contour map of the $K$-band total intensity image with polarization vector lines. Our polarimetric data identified an elongated region with small polarizations lying at a position angle of $-45^{\circ}$ and a polarization vector alignment on the east side of the central star. 


\section{Radiative transfer modeling}

We have modeled the bipolar nebula of Frosty Leo by means of radiative transfer calculations. We, for the first time, attempted to reproduce the spectral energy distribution (SED), the total intensity images and the polarization maps of this object. We used a recently developed Monte Carlo code (Ohnaka et al. 2006). We have modified the original code to use the method proposed by Fischer et al. (1994) to determine the direction of the scattered light, which takes into account the polarization status of the incident light.

The dust grain model assumes a silicate spherical core (Draine 1985) with a water ice mantle (Bertie et al. 1969) and large grains $(0.1 \leqslant a \leqslant 2.0 \mu \mathrm{m}$; cf. Lopez et al. 2001) in the disk and small grains $(0.05 \leqslant a \leqslant 0.8 \mu \mathrm{m}$; cf. Dougados et al. 1990). The fraction of the water ice in a grain is $50 \%$ in volume. The model geometry has an axisymmetric structure with an equatorial dust condensation (Kahn \& West 1985).

Figure 2 shows the result of the numerical simulations. The adopted model parameters are an optical depth at $2.2 \mu \mathrm{m}$ of 6 , an equator-to-pole density ratio of 10 , an inner radius of $4000 R_{*}$, and an outer radius of $57,000 R_{*}$.

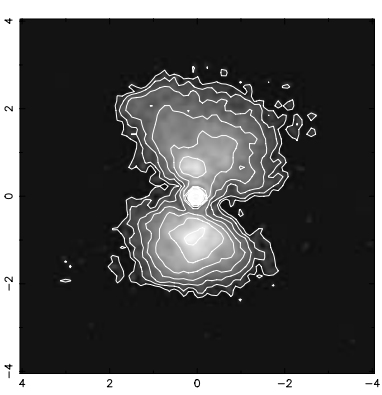

offset R.A. (orcsec)

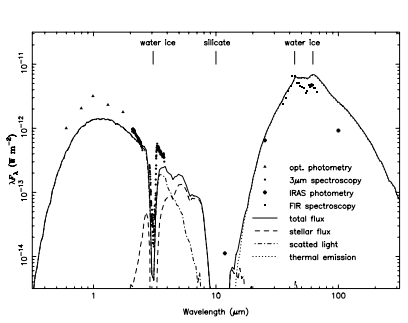

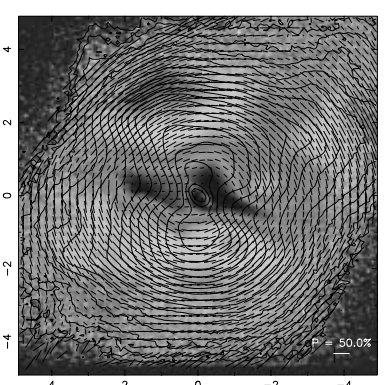

offset R.A. (orcsec)

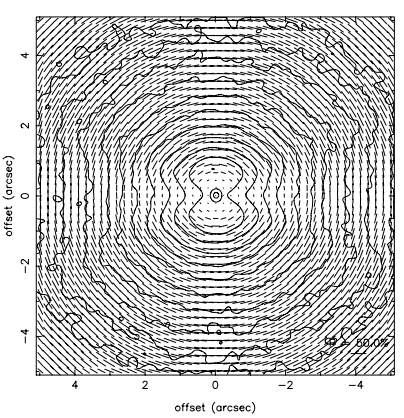

Figure 1: The left panel shows the $K$-band speckle image with contour lines plotted in increments of $0.5 \mathrm{mag}$. The angular resolution is $0 . " 188$. The right panel displays the total intensity with contour lines, polarization image in gray scale, and polarization vector lines.

Figure 2: The model SED compared with the observed SED (left). The $K$ band model image with polarization vectors (right).

\section{References}

Bertie, J. E., Labbé, H. J., \& Whalley, E. 1969, J. Chem. Phys. 50, 4501

Draine, B. T. 1985, ApJS 57, 587

Fischer, O., Henning, Th., \& Yorke, H. W. 1994, A\&A 284, 187

Kahn, F. D., \& West, K. A. 1985, MNRAS 212, 837

Lopez, B., Tuthill, P. G., Danchi, W. C., Monnier, J. D., \& Niccolini, G. 2001, A\&A 377, 90

Ohnaka, K., Driebe, T., Hofmann, K.-H., et al. 2006, A\& $A$ 445, 1015

Omont, A., et al. 1990, ApJ 355, L27

Roddier, F., Roddier, C., Graves, J. E., \& Northcott, M. J. 1995, ApJ 443, 249

Rouan, D., Omont, A., Lacombe, F., \& Forveille, T. 1988, A\&SA 189, L3

Sahai, R., Bujarrabal, V., Castro-Carrizo, A., Zijlstra, A. 2000, A\& A 360, L9

Weigelt, G. 1977, Opt. Comm. 21, 55 